\title{
A control and monitor system for the liquid argon cryogenics of the calorimeter of the ATLAS detector
}

\author{
M. Chalifour, ${ }^{a}$ O. Dalifard, ${ }^{b}$ C. Eder, ${ }^{b}$ E. Jules, ${ }^{b}$ T. Roulet ${ }^{b}$ and F. Wicek ${ }^{\text {b冈 }}$ \\ ${ }^{a}$ CEA/Saclay - 91191 Gif-sur-Yvette, France \\ ${ }^{b}$ LAL, Université Paris-Sud CNRS/IN2P3, 91898 Orsay, France \\ E-mail: wicek@lal.in2p3.fr
}

\begin{abstract}
In a few weeks the Large Hadron Collider which is built at CERN will deliver its first beam. ATLAS (A Toroïdal Large hadron collider ApparatuS [1]] is one of the four experiments whose aim is to analyze the products of the collisions. As many of these particle detectors, ATLAS is designed with four concentric layers which are, from inside to outside: the inner detector to measure the charged particles tracks, the electromagnetic calorimeter to measure the energy deposited by both electrons and photons, then the hadronic calorimeter and finally the muon detector. The active part of the electromagnetic calorimeter is a bath of liquid argon [2] whose large volume $\left(78 \mathrm{~m}^{3}\right)$ has to be maintained precisely at its operating temperature (about $88 \mathrm{~K}$ ) by the means of nitrogen circulation. In this paper we will describe how with industry-like control systems an application has been built for the command and the monitoring of the whole cryogenics equipment.
\end{abstract}

KEYWORDS: Calorimeters; Detector control systems (detector and experiment monitoring and slow-control systems, architecture, hardware, algorithms, databases); Detector cooling and thermo-stabilization; Control and monitor systems online.

\footnotetext{
* Corresponding author.
} 


\section{Contents}

\begin{tabular}{|ll}
\hline 1. Introduction & 1 \\
\hline
\end{tabular}

\begin{tabular}{|lr}
\hline 2. The services & 2 \\
\hline
\end{tabular}

\begin{tabular}{lr|}
\hline 3. The clients & $\mathbf{4}$ \\
\hline 3.1 Standby mode & 4 \\
\hline 3.2 Purge & 4 \\
\hline 3.3 Cool-down & 5 \\
\hline 3.4 Filling & 6 \\
\hline 3.5 Normal regulation & 6 \\
\hline 3.6 Emptying & 6 \\
\hline 3.7 Warming-up & 6 \\
\hline
\end{tabular}

\begin{tabular}{|lr|}
\hline 4. Realization & 7 \\
\hline 4.1 The field layer & 7 \\
\hline 4.2 The control logic & 8 \\
\hline 4.3 The supervision layer & 9 \\
\hline
\end{tabular}

5. Conclusion

\section{Introduction}

The liquid argon control and monitoring process is based upon a "client/supplier" architecture. A client is a consumer of cooling power, whereas the supplier produces and distributes the cooling power. The five clients are composed of the three cryostats, which contain the three calorimeters, as well as the two liquid argon storage tanks dedicated to the cryostats (one for the barrel and one for both end-caps). The supplier controls the resources (the cryogenic equipment related to the nitrogen storage and circulation). The control process was drafted according to a functional analysis and designed with four or five hierarchical levels, depending on the complexity of the structure.

Calorimeters are fragile detectors, from a mechanical as well as from an electrical point of view. Besides the building effort, and taking into account the cryogenics command and monitoring specificity, physicists and engineers planned a very precise mechanical constraints protocol in order to bring the detectors to their operating temperature $(88.3 \mathrm{~K})$ and to maintain this temperature during the whole duration of the experiment (about fifteen years).

In order to satisfy these constraints, a redundant scheme of the frigorific fluid distribution was designed. 


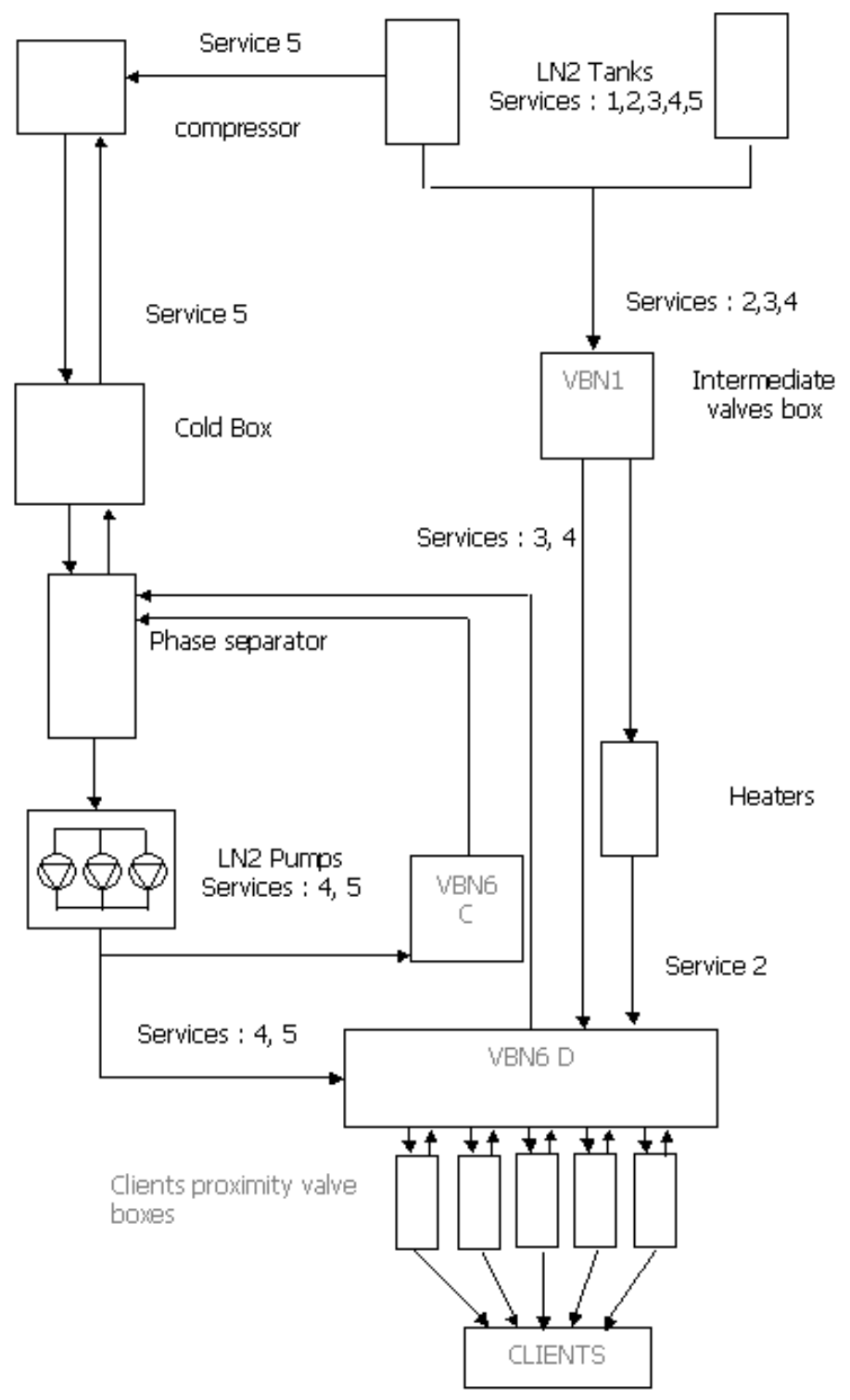

Figure 1. Services and distribution functional layout.

\section{The services}

The nitrogen storage and circulation - the supply function - are split into five operating modes which are called "services". Each service uses a subset of the cryogenics equipment (tanks, distribution boxes, pumps, heaters). This partition allows each element to be turned ON one after the other, beginning with the nitrogen storage tanks alone (service 1) and ending by turning $\mathrm{ON}$ the refrigerator (service 5). Shifting from one service to the next one is systematically operated manually. However in case of a failure of one of the elements the control system can return to a lower level service automatically.

The first service is a basic service for the distribution initialisation and the liquid nitrogen storage management. It manages the availability of the two liquid nitrogen tanks (Figure 1 , "LN2 Tanks). 
The second service is dedicated to the cooling of the calorimeters. Taking into account the mechanical constraints, the cooling operation consists of the vaporization of the liquid nitrogen coming from the storage tanks inside a nitrogen/thermalized water heat exchanger. Then this flow is warmed up by a heater whose temperature setup follows a fixed cool-down ramp calculated by a Programmable Logic Controller (PLC). An interlock managed by the Detector Control System (DCS) can stop the ramping process by stopping the cool-down ramp in order to by-pass the exchanger flow until cooling authorization is given again.

The third service is the first one which is dedicated to the liquid nitrogen distribution. It is the back-up service for the next two services in situations where they would be out of order due to a maintenance operation or a failure. This service distributes liquid nitrogen coming from the surface-located storage tanks to the calorimeters heat exchangers through intermediate distribution boxes (Figure 1 "VBN1" and "VBN6 D"). The proximity distribution boxes control the pressure, flow and liquid quality meter of the nitrogen distribution. This service can only be used temporarily and manual operator intervention is necessary to set the process into one of the next two services.

The fourth service is the first one which controls the calorimeter cryogenics in a continuous way. It consists of the transfer of the liquid nitrogen from the tanks to the cryostats through the intermediate distribution box (Figure 1, "VBN1") and a phase separator where both pressure and level are regulated. The liquid transfer is assisted by means of three circulation pumps (Figure 1. "LN2 Pumps") in redundancy mode (only one pump is powered at a time), allowing a constant flow and pressure regulation. Below the service management distribution box (Figure 1] "VBN6-D") the flow path is identical to the previous service.

The fifth service is the liquid distribution service which is used by default. A $25 \mathrm{~kW}$ (at $84 \mathrm{~K}$ ) nitrogen refrigerator is connected to the phase separator and to the circulation pumps. The compressor is located at the surface and the cold box is located in the service cavern.

Figure 1 represents a schematic view of the distribution functional layout: It shows the services associated to the cryogenic equipments. The VBN6-D distribution box is connected to the nitrogen tanks through the intermediate VBN1 distribution box directly (service 3), or with the heaters (service 2), with the use of the phase separator, pumps and the VBN6-C circulation box (services 4), and with the use of the cold box and the compressor (service 5). Clients are connected to the VBN6-D distribution box through the clients proximity valve boxes.

In addition to these five services and their associated equipment, the resources include the insulation vacuum system which separates the "cold" inside wall of the calorimeter from the outside wall. Each of the three calorimeters makes use of one rotary vane pump $\left(60 \mathrm{~m}^{3}\right.$ per hour), one roots pump ( $250 \mathrm{~m}^{3}$ per hour) and one oil diffusion pump (two for the barrel cryostat $-2000 \mathrm{l} / \mathrm{s}$ ) in order to obtain a vacuum insulation better than $10^{-7}$ bar.

The front-end calorimeter electronics is located outside the cryostat, and there are special feedthroughs which enable the connection of the detector electrodes to the electronic boards. The vacuum of these feedthroughs is managed by the control and monitoring system too, and the temperature is regulated to be about $20^{\circ} \mathrm{C}$ with small heaters to avoid condensing water inside the feedthroughs. Each of the end cap cryostats has 28 feedthroughs and the barrel has 64 feedthroughs (32 on each side). In order to minimize the number of analog outputs issued from the PLC (far more expensive than the digital ones), the result of the temperature regulation computation (12 bits) is sent to an extra box which realizes the digital to analog conversion and the power amplification. 


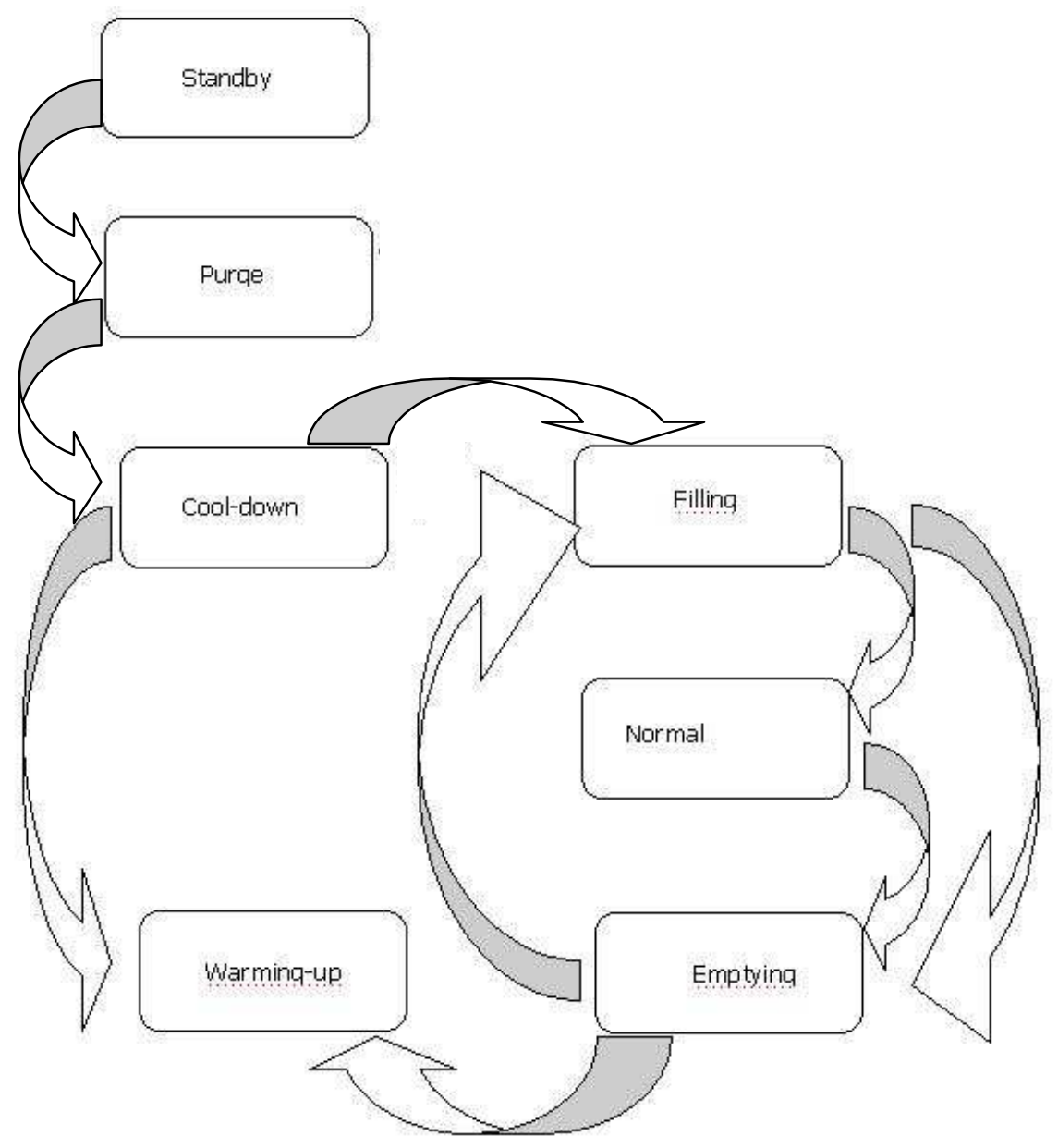

Figure 2. Modes Flow diagram for one client.

\section{The clients}

For each of the clients several sequential operations or "modes" have been defined. Figure 2 represents the flow diagram of the modes for one of the clients (cryostats). A client dedicated table is created in the software process to enable or disable the transition from a mode to another one depending on the present state of all the clients.

\subsection{Standby mode}

The system is warm (room temperature) and no regulation is set to be active by this mode but some actuators can be operated manually for tests (valves, heaters, vacuum pumps) while others will be blocked (filling the cryostat for instance).

\subsection{Purge}

Purge is an automatic process for the three cryostats, and a manual operation for the two argon tanks. Before cooling the three calorimeters, it is necessary to remove all the contaminants present in the cryostats or calorimeters. This is done by pumping and then filling the cryostats 


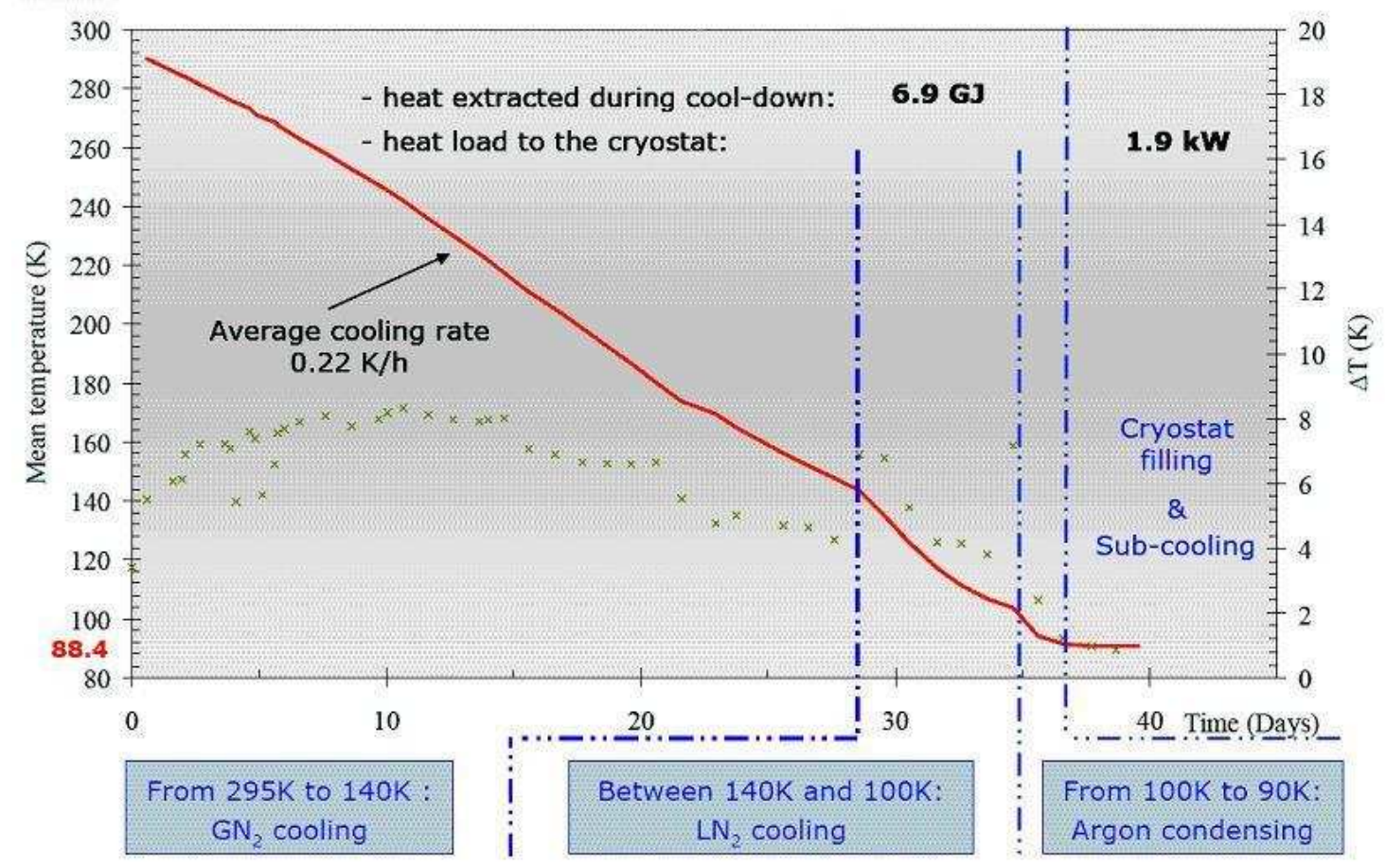

Figure 3. Barrel temperature during cool-down (Plot C. Fabre, CERN, [3]].

with clean and dry argon for the barrel and clean and dry helium for the two end-caps (the necessity of using helium will be explained below). These operations are repeated until the remaining traces of air and water in the argon or helium contained in the cryostats correspond to a purity better than $10 \mathrm{ppm}$ of equivalent $\mathrm{O}_{2}$. At the end of the purge, the control system gives an "end-mode" signal that means that the client table can be updated.

\subsection{Cool-down}

By cryogenics construction it is possible to cool-down only one calorimeter at a time. The system is able to go to the cool-down mode just after a purge or a warming-up.

The three cryostats and the three calorimeters are made of materials with different thermal coefficients. Therefore small temperature discrepancies may induce unacceptable constraints or displacements. For the barrel seven temperatures and eight displacements criteria are defined to form a common interlock stopping the cooling process; for each end-cap eleven temperatures criteria are defined.

Thermal models have been made, by decomposing the calorimeters in several subsets to estimate a reasonable power exchange in a free convection mode. A temperature difference of $40 \mathrm{~K}$ between the input and the output of the heat exchangers satisfies the above displacements criteria. This temperature gap provides the cooling power. It allows to extract the 7 Gigajoules of the barrel at a speed of $0,22 \mathrm{~K} / \mathrm{h}$ Figure 3 with gaseous argon as heat transfer fluid as well as to extract the 16 Gigajoules of each end-cap at the speed of $0,13 \mathrm{~K} / \mathrm{h}$ with gaseous helium as heat transfer fluid (it would take too much longer with argon gas).

Starting from ambient temperature (about $290 \mathrm{~K}$ ) the cooling operation is achieved by a forced circulation of cold gaseous nitrogen (Figure $3 . \mathrm{GN}_{2}$ cooling) in the heat exchangers of the calorimeters ( 4 for the barrel, 2 for each end-cap). By adjusting the temperature of the incoming cold nitrogen with a ramp adapted to the criteria defined above and the flux by 
adjusting the differential pressure, the barrel cryostat is filled with gaseous argon maintained at a pressure of 1.3 bar. The two end-caps are filled with gaseous helium and also maintained at a pressure of 1.3 bar. At about $110 \mathrm{~K}$, the power exchange becomes ineffective (the cooling rate decreases dramatically), therefore pressurized liquid nitrogen is circulated in the heat exchangers instead of gaseous nitrogen (Figure $3 \mathrm{LN}_{2}$ cooling). This is done by changing the service. When the temperature of about $90 \mathrm{~K}$ is reached we consider that the cool-down operation is finished. The end-cap cryostats are purged with argon gas and the system updates the table of the modes: it authorizes the filling mode or the warming up mode.

\subsection{Filling}

The cryogenics system can only fill one calorimeter at a time. The order in which to fill is chosen by the operator manually. The table of the modes eventually authorizes the operation.

The filling operation is done by condensing and transferring liquid argon from the argon storage tank to the bottom of the cryostat (Figure 3 argon condensation). The heat exchangers in the cryostats work with the inlet valve regulating the liquid nitrogen flow (based on a temperature set point) and the outlet valve regulating the liquid nitrogen pressure. Obviously, the barrel tank has to be filled before filling the barrel cryostat, and the end-caps storage tanks have to be half-full at least before filling one of the end-cap cryostats. Moreover entering filling mode is disabled when one of the clients is still in this mode. Filling mode is finished when the level in the expansion vessel is above $40 \%$.

After filling one can change to the normal regulation mode or the emptying mode.

\subsection{Normal regulation}

The expansion vessel heat exchanger regulates the argon gaseous pressure. The inlet valve of the heat exchanger regulates this pressure at 1.25 bar, while the outlet valve of the heat exchanger regulates the pressure inside the heat exchanger at 3 bar. The heat exchanger in the liquid argon volume of the cryostat regulates the temperature of the argon bath. The inlet valve regulates the liquid nitrogen flow, based on a quality meter $(50 \%)$, while the outlet valve regulates the nitrogen pressure inside the heat exchanger at 3 bar.

After this mode one can only change to the emptying mode.

\subsection{Emptying}

The argon storage tanks are situated such that the cryostats content can be dumped into it by gravity. In normal situation, the liquid argon pumps will be used to empty the cryostats to speed up the process to 5000 liter per hour. The pressure in the cryostat (and thus in the expansion vessel) should be regulated so that it will never fall below 1.5 bar. Following the same principle than the filling mode, the barrel tank must be empty before emptying the barrel cryostat, and the end-caps storage tank has to be half-full at most before emptying one of the end-cap cryostats. Moreover entering emptying mode is disabled when one of the clients is still in this mode.

After this mode one can only change to the filling mode or the warming-up mode.

\subsection{Warming-up}

Electrical heaters are used to warm up the cryostat. These heaters have an "ON/OFF" regulation controlled by the control system. As for the cool-down operation, the control system regulates the warm-up of the cryostat with a constant slope $(0,22 \mathrm{~K} / \mathrm{h}$ for the barrel and $0,13 \mathrm{~K} / \mathrm{h}$ for both end-caps). 
Table 1. Field layer distribution.

\begin{tabular}{|l|l|l|l|}
\hline $\begin{array}{l}\text { Board } \\
\text { Type }\end{array}$ & $\begin{array}{l}\text { Boards } \\
\text { number }\end{array}$ & $\begin{array}{l}\text { Number of channels } \\
\text { per board }\end{array}$ & $\begin{array}{l}\text { Total channels } \\
\text { number }\end{array}$ \\
\hline DI & 40 & 32 & 1280 \\
\hline DO & 23 & 32 & 736 \\
\hline AI & 84 & 8 & 672 \\
\hline AO & 18 & 4 & 72 \\
\hline
\end{tabular}

Although this mode will be processed at the very end of the experiment lifetime, the whole life cycle has been already processed once for each one of the three detectors, in the facility room where the detectors have been assembled. The differences between this test and the real working condition in the cavern are the lack of the refrigerator equipment, the absence of radiation (but argon was chosen because of its radiation immunity) and the normal regulation mode duration, reduced to several weeks for the test.

\section{Realization}

In order to elaborate a collaborative project between various equipment groups for a single control system for all LHC cryogenics equipments (machine, experiment cryostats or magnets) a framework named UNICOS (Unified Industrial Control System [4]) was developed by CERN. This normalization system was not created from scratch but based on generic software architecture evolved from the LEP [5] cryogenics experiment. The use of the UNICOS specification was obvious to us since we were in charge of developing the control and monitoring system for the electromagnetic calorimeter of the ATLAS experiment. It was also the best way to integrate our cryogenic control system within the LHC operational environment.

UNICOS is a framework developed to produce control and monitoring applications for three-layer control systems [6]

- The field layer contains the process sensors and actuators connected to the control system via Inputs/Outputs (I/0) boards or field buses.

- The control logic is implemented in the control layer (the PLCs).

- The supervision layer provides operators with monitoring and command facilities by means of supervision control and data acquisition (SCADA) systems.

A data server acts as the gateway between the control logic implemented on multiple PLCs and the supervision. A software layer named "middleware" is deployed on the different devices in order to manage the communication of the data.

\subsection{The field layer}

The field layer contains process equipment such as ON/OFF valves, heaters or pumps, driven by a digital signal and digital sensors like the end position switches of the ON/OFF valves. It contains analog actuators such as control valves and analog sensors (temperature, pressure, control valves position feedback). The digital and analog sensors are connected to the control system by Digital Input (DI) and Analog Input (AI) channels respectively. Likewise the $\mathrm{ON} / \mathrm{OFF}$ and analog actuators are connected to the control system by Digital Output (DO) and Analog Output (AO) channels respectively. The field layer distribution is showed on Table 1.

These I/O boards are assembled into 24 racks, and linked to the physical elements of the field layer by 2,450 connectors and 95 kilometers of cable. 
Moreover, in order to reduce the number of I/O connections, some complex equipment of the field layer is linked to the control logic trough a Profibus field bus:

- The three pumps of the nitrogen distribution and both argon pumps are built with an asynchronous motor which is driven by a frequency variator controlled though a Profibus link;

- All the vacuum pressure sensors (Pirani [7] or Penning [7] type) are Profibus connected too.

- The compressor of the refrigerator equipment and an autonomous Siemens PLC which manages the turbine are connected to the main control logic by a Profibus link.

\subsection{The control logic}

Nowadays many industrial control system components are used in the high energy physics community. The UNICOS framework proposes the use of industry-like PLCs. For our purpose the "Modicon Quantum" modules from "Schneider-Electric-Télémécanique" were selected. The unit controller (140CPU534) is based on a 80586 microprocessor clocked at $133 \mathrm{MHz}$ [8], This is no longer the state-of-the-art architecture we would find today but it fitted our needs when the choice was made in the early 2000s and now, as we have completed the software integration, we can say that this choice is still perfectly convenient. The whole embedded software is split into 6 unit controllers:

- One controller is devoted to the nitrogen circulation (including the pumps thanks to a Profibus extension). It supports the "high-level" software, in particular the services management.

- One controller is devoted to each one of the three clients. The modes which define the present state of the client and the rules which enable or disable the entering in a particular mode are managed by this controller. All the vacuum pressure sensors (insulation and feedthroughs) are seen by this controller through a Profibus link. The heating of the feedtroughs is controlled by this logic too.

- Two controllers are devoted to the refrigerator equipment.

All the controllers are located in a dedicated room, close to the service room which contains the cryogenic equipment, and approximately 50 meters beneath the surface, with the exception of the one devoted to the compressor of the refrigerator, which is located at the surface.

Inside a PLC, the UNICOS framework gives us the rules to break down the process into a hierarchy of modules:

- At the top of the process we can find the Process Control Objects (PCO) which represent the control logic associated to a sub-equipment of the cryogenic process (for instance the phase separator, a valve box, the nitrogen tanks,...).

- Below the PCO we have an image of all the field objects which belong to the subequipment (valves, pumps, heaters) and the process itself (usually a "grafcet" [9] although UNICOS doesn't provide such a toolkit) which controls all these elements, considering the value of the sensors associated to the process and the whole enabling or disabling conditions transmitted in a hierarchical way. At this level we can find controller objects encapsulating a regulation algorithm based on the PID (Proportional-IntegrationDerivation) model. The controller object is always associated to an analog field object .

- A the very bottom we can find all the I/O objects that represent the electrical elements. In the particular case of the AI element, it executes the conversion between a physical value received from the sensor (temperature, pressure) to an engineering value to be used by the control logic. The AO element must guarantee the conversion of the control system orders to the field actuators. 


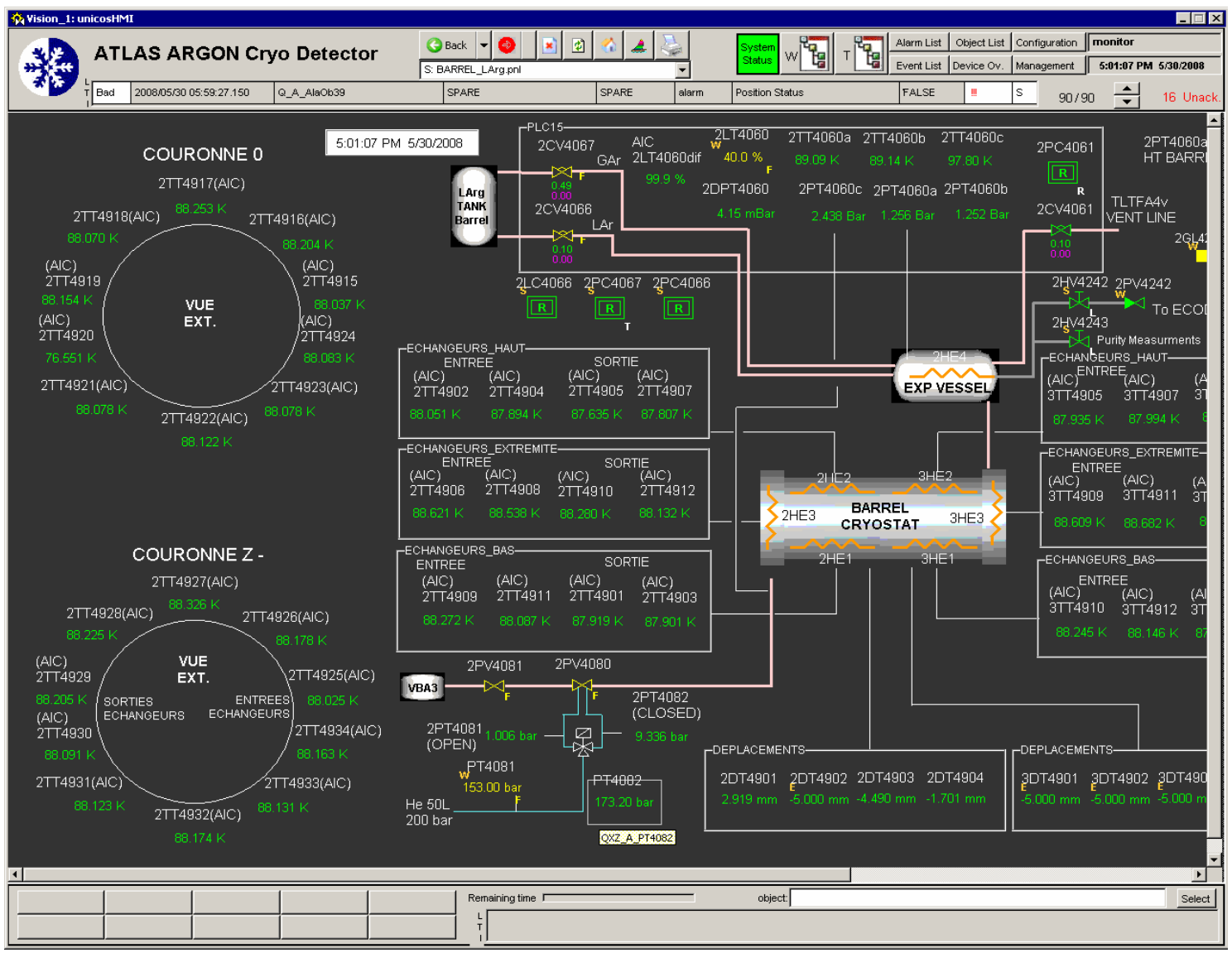

Figure 4. One out of a hundred of supervision views.

\subsection{The supervision layer}

The supervision layer provides operators with monitoring and command facilities by means of Supervision Control And Data Acquisition systems (SCADA). The Austrian product "PVSS II" [10], already used at CERN by physicists, was chosen by the UNICOS team who developed a proprietary tool which automatically generates the files needed by the SCADA by processing the complete list - the database - of parameters of each I/O, field object and PCO. In fact the resulting file is implemented in the data server. Moreover the UNICOS tool generates all the basic level objects for the PLC embedded application and the middleware is automatically configured.

Nevertheless our cryogenic application requires almost a hundred of supervision views, each one representing the synoptic drawing of a sub-system with all the indicators that might be needed by the operator to monitor the process (Figure 4. These views follow the hierarchical structure of the application but no code development is required for the production of the supervision layer.

\section{Conclusion}

For each of the three cryostat parts, a complete life-cycle (e.g. the sequential operations as listed in Figure 2 was performed during years 2004-2005 in the surface-based facility where the detectors were assembled. As soon as the general input/output electrical tests were performed, our slow control system was ready to proceed with the rinsing, cooling (6 to 10 weeks duration), filling, normal run, emptying and warm-up (6 to 10 weeks duration too) operations for each of the three 
cryostats, allowing us to validate both hardware installation and software application. These validation tests were particularly important for the ones which follow the normal run mode, because these operations will normally never occur again before the end of the instrument life. There were no differences between these validation tests and the real working conditions except for the lack of the refrigerator equipment.

After a complete test, the cryostat was brought down in its definitive location inside the ATLAS cavern. The de-cabling and re-cabling of the whole electrical and cryogenic installation made a new input/output general test mandatory.

One at a time each of the three cryostats was purged, cooled down and filled with liquid argon again. These operations were performed during 2006 and the first six months of the 2007. Meanwhile we integrated the refrigerator into the process and nowadays all three cryostats are in normal run mode, filled with $90 \mathrm{~m}^{3}$ of quiet and cold liquid argon whose temperature is regulated better than $0.1 \mathrm{~K}$ around the ideal working point.

\section{Acknowledgment}

We thank Johan Bremer, who is the team leader of the cryogenics installation for the liquid argon calorimeter of ATLAS in CERN, Caroline Fabre in CERN, Patrick Cornebise and the cabling team from the "Service Electronique" of the LAL, who were in charge of all the hardware interconnection cabling operations in CERN. We thank Francoise Maréchal at LAL and R.D. Schaffer at CERN, for the reading and correction of this paper.

\section{References}

[1] ATLAS collaboration, The ATLAS Experiment at the CERN Large Hadron Collider, 2008 IINST 3 S08003.

[2] ATLAS collaboration, ATLAS Liquid Argon Calorimeter: Technical Design Report, CERNLHCC-96-041, 15 December 1996

[3] C. Fabre, Cryogenics for Liquid Argon Calorimeter, in Symposium for the inauguration of the LHC Cryogenics, Geneva, 31 May to 01 June 2007.

[4] P. Gayet, Industrial Controls \& Unicos, in LHC-CP workshop 3 Session 3, 18 March 2002.

[5] LEP: the Large Electron Positron collider, CERN-ARCH-LEP-01-001 to 06-017

[6] Ph. Gayet and R. Barillère, Unicos A framework to build industry like control systems: Principles \& Methodology, ICALEPS Int. Conf. On Accelerator \& Large Experiment Physics Control Systems. Geneva, 10 - 14 Oct 2005, WE2.2-61 (2005).

[7] Adixen (Alcatel Vacuum technology), Vacuum measurement, http://www.adixen.nl/downloads/GAUGES_100.pdf.

[8] Modicon TSX, Quantum Automation Series Hardware reference Guide, Version 6.0, Schneider Electric, http://www.schneider-electric.fr.

[9] Grafcet: GRAphe Fonctionnel de Commande Etapes/Transitions, IEC 60848:2002, http://www.iec.ch.

[10] PVSS (Prozessvisualisierungs- und Steuerungs-System), ETM Professional control GmbH, Kasernenstrasse 29, A 7000 Eisenstadt Austria (Web: http://www.pvss.com). 